\title{
Force-Dependent Fragility in RNA Hairpins
}

\author{
M. Manosas, ${ }^{1}$ D. Collin, ${ }^{2}$ and F. Ritort ${ }^{1}$ \\ ${ }^{1}$ Departament de Física Fonamental, Facultat de Física, Universitat de Barcelona Diagonal 647, 08028 Barcelona, Spain \\ ${ }^{2}$ Merck \& Co., Inc., Automated Biotechnology Department, North Wales, Pennsylvania 19454, USA \\ (Received 23 September 2005; published 31 May 2006)
}

\begin{abstract}
We apply Kramers theory to investigate the dissociation of multiple bonds under mechanical force and interpret experimental results for the unfolding and refolding force distributions of an RNA hairpin pulled at different loading rates using laser tweezers. We identify two different kinetic regimes depending on the range of forces explored during the unfolding and refolding process. The present approach extends the range of validity of the two-states approximation by providing a theoretical framework to reconstruct freeenergy landscapes and identify force-induced structural changes in molecular transition states using single molecule pulling experiments. The method should be applicable to RNA hairpins with multiple kinetic barriers.
\end{abstract}

Single molecule pulling experiments allow one to exert mechanical force on individual molecules such as nucleic acids, proteins, and macromolecular complexes [1]. By recording force-extension curves it is possible to determine the free energies and kinetic parameters of biomolecules and search for intermediates and pathways in biochemical reactions. Over the past years single molecule techniques have been successfully applied to investigate the breakage of molecular bonds in many biological systems such as proteins [2], DNA molecules [3], RNA molecules [4], ligand-receptor binding [5], and beyond, e.g., metallic gold nanowires stretched with an atomic force microscope [6]. Under mechanical load all these structures yield at different values of the applied force in a dynamical process that is stochastic and loading rate dependent. The study of breakage forces under nonequilibrium conditions is known as dynamic force spectroscopy [7]. A detailed comprehension of the rupture kinetics of biomolecular complexes has implications in our understanding of their kinetic stability which is important in enzymatic and/or regulatory processes.

Here we investigate the unfolding and refolding kinetics of RNA hairpins using laser tweezers [4,8]. The RNA sequence and its native structure are shown in Fig. 1. To manipulate the RNA hairpin, two beads are attached to the ends of the RNA hairpin by inserting two hybrid RNA and DNA handles [9]. One of the beads is immobilized on the tip of a micropipette, while the other bead is captured in the optical trap. By moving the micropipette a force is exerted upon the ends of the RNA hairpin and the force-extension curve (FEC) recorded. In Fig. 1 we show the experimental FEC corresponding to a complete cycle of a ramping process where the force is first raised and relaxed afterwards. In the pulling process the molecule is initially in its native folded structure, and the force is increased at a certain rate $r$ until the molecule unfolds. If the process is reversed, i.e., the force is decreased at rate $-r$, the molecule folds back again (relaxing process) [10]. The unfold- ing and refolding of the molecule can then be identified as force-extension jumps observed in the FEC. By repeatedly pulling the molecule many times we obtain the distribution of unfolding (refolding) forces, i.e., the force at which the first unfolding (refolding) event occurs along the pulling (relaxing) process (Fig. 1). The experimental distribution of the unfolding $(\mathrm{u})$ and refolding $(\mathrm{r})$ forces $\rho_{\mathrm{u}(\mathrm{r})}(f)$ at different loading rates $r$ is shown in the inset of Fig. 1 .

To model the hairpin we follow Cocco et al. [11] and restrict the number of configurations of an $N$ base-pair (bp) RNA hairpin to the set of configurations where the first $n$ bps are opened and the last $N-n$ are closed (the total

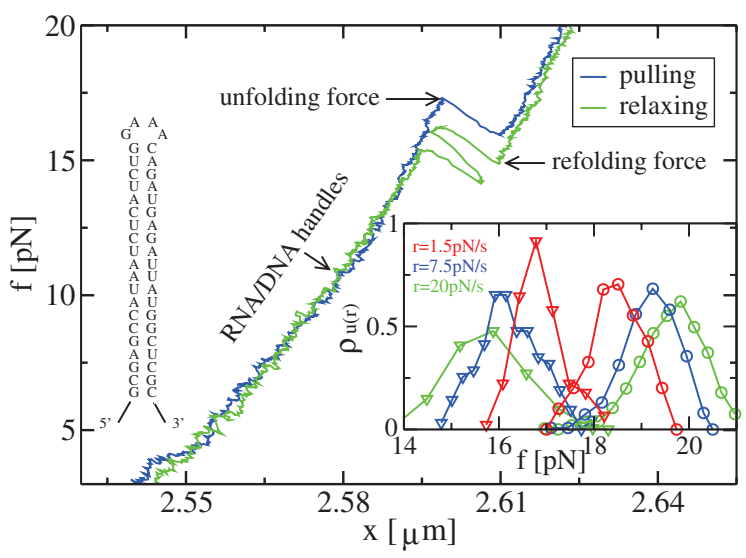

FIG. 1 (color). Experimental FEC for the RNA hairpin in a ramping cycle where the force is first increased and after decreased at a rate of $r=3 \mathrm{pN} / \mathrm{s}$, the blue and green curves representing the pulling and relaxing processes, respectively. The arrows indicate the unfolding and refolding forces, which correspond to the first unfolding and refolding events. The first part of the FEC corresponds to the stretching of the RNA and DNA hybrid handles. Inset: Experimental distribution of the unfolding (circles) refolding (triangles) forces. Number of pulls: 129,385 , and 703 for $r=1.5,7.5$, and $20 \mathrm{pN} / \mathrm{s}$ (red, blue, and green), respectively. 
number of configurations being $N+1$ ). The end-to-end distance is a well-defined reaction coordinate for the unfolding and refolding reaction. We use the variable $n$ to label the state of the hairpin; e.g., the folded (F) state corresponds to $n=0$ and the unfolded (UF) state to $n=$ $N$. The stability of each state $n$ depends on its free energy, $G_{n}(f)$, at a given applied force $f$ [11],

$$
G_{n}(f)=G^{0}(n)+g(n, f),
$$

where $G^{0}(n)$ is the free energy of formation at zero force and $g(n, f)$ is the force-dependent contribution to the free energy. The latter is given by $g(n, f)=G_{\mathrm{ss}}(n, f)-$ $f x_{n}(f)$, where the functions $x_{n}(f)$ and $G_{\mathrm{ss}}(n, f)$ are the end-to-end distance and the entropic correction to the free energy of an $M$-bases long single-stranded RNA (ssRNA) at force $f, M$ being the number of bases released after the opening of $n$ bps. The latter can be computed as the reversible mechanical work needed to stretch the ends of an $M$-bases long ssRNA a distance $x_{n}(f)$,

$$
G_{\mathrm{sS}}(n, f)=\int_{0}^{x_{n}(f)} F_{\mathrm{sSRNA}}(x) d x,
$$

where $F_{\text {ssRNA }}(x)$ is the FEC of the ssRNA [12]. The freeenergy landscape of a hairpin, $G_{n}(f)$, as a function of $n$ is known to be rugged with different kinetic barriers (or transition states) depending on the sequence and on the applied force $f$ [14]. We use the Mfold prediction [15] to extract the free energy of the molecule at zero force, $G^{0}(n)$. In what follows we take the $\mathrm{F}$ state as the reference state for the free energy, i.e., $G_{n=0}(f)=G^{0}(n=0)=0$.

The kinetics of unfolding (i.e., the transition between the $F$ and the UF states) is an activated process with a forcedependent effective barrier, $B_{\text {eff }}(f)$, measured relative to the $\mathrm{F}$ state. The rates of unfolding and refolding, $k_{\mathrm{u}}(f)$ and $k_{\mathrm{r}}(f)$, can be computed as the first passage rates [16] for a Brownian particle to cross a force-dependent effective barrier $B_{\text {eff }}(f)$ :

$$
k_{\mathrm{u}}(f)=k_{0} e^{-\beta B_{\text {eff }}(f)}, \quad k_{\mathrm{r}}(f)=k_{0} e^{-\beta\left[B_{\text {eff }}(f)-G_{N}(f)\right]},
$$

where $G_{N}(f)$ is the free-energy difference between the $\mathrm{F}$ and UF states at force $f, \beta=1 / k_{B} T$ with $k_{B}$ and $T$ being, respectively, the Boltzmann constant and the bath temperature, and $k_{0}$ is an attempt frequency. An analytical expression for $B_{\text {eff }}(f)$ can be derived from Kramers theory applied to the dissociation of consecutive bonds under mechanical force in the stationary approximation $[7,16]$,

$$
B_{\mathrm{eff}}^{\mathrm{KT}}(f)=k_{B} T \ln \left[\sum_{n=0}^{N} h(n) e^{\beta G_{n}(f)}\right],
$$

with $h(n)=\sum_{n^{\prime}=0}^{n} e^{-\beta G_{n^{\prime}}(f)}$. The variation in force of the effective barrier gives information about its position along the reaction coordinate:

$$
x_{\text {eff }}^{\mathrm{F}}(f)=-\frac{\partial B_{\text {eff }}(f)}{\partial f}, \quad x_{\text {eff }}^{\mathrm{UF}}(f)=\frac{\partial\left[B_{\text {eff }}(f)-G_{N}(f)\right]}{\partial f},
$$

where $x_{\text {eff }}^{\mathrm{F}}(f)$ and $x_{\text {eff }}^{\mathrm{UF}}(f)$ are the distances from the effective barrier to the $\mathrm{F}$ and UF states, respectively. The location of the barrier along the reaction coordinate is related to the fragility of the molecule which determines how much the unfolding and refolding kinetics is sensitive to the force. To characterize the fragility we introduce the parameter $\mu$ defined as

$$
\mu(f)=\frac{x_{\mathrm{eff}}^{\mathrm{F}}(f)-x_{\mathrm{eff}}^{\mathrm{UF}}(f)}{x_{\mathrm{eff}}^{\mathrm{F}}(f)+x_{\mathrm{eff}}^{\mathrm{UF}}(f)} ;
$$

$\mu<0$ corresponds to a brittle structure, e.g., the case of hairpins stabilized by tertiary contacts where the barrier is located near to the $\mathrm{F}$ state, whereas $\mu>0$ represents a flexible or compliant structure, i.e., molecules that can easily deform under applied force and the barrier is close to the UF state [17].

The rates (3) are related to the force distributions by the expression $\rho_{\mathrm{u}(\mathrm{r})}(f)=\frac{k_{\mathrm{u}(\mathrm{r})}(f)}{r} \exp \left[-(+) \int_{f^{\prime}}^{f} \frac{k_{\mathrm{ur}(\mathrm{r})}(y)}{r} d y\right]$ with $f^{\prime}$ being the initial force in the pulling (relaxing) process [18]. The unfolding (refolding) rates read as $k_{\mathrm{u}(\mathrm{r})}(f)=$ $\rho_{\mathrm{u}(\mathrm{r})}(f) \frac{1}{r P_{\mathrm{u}(\mathrm{r})}(f)}$, where $P_{\mathrm{u}(\mathrm{r})}(f)$ is the probability that the molecule remains in the F (UF) state along the pulling (relaxing) process until reaching the force $f, P_{\mathrm{u}(\mathrm{r})}(f)=$ $+(-) \int_{f^{\prime}}^{f} \rho_{\mathrm{u}(\mathrm{r})}(y) d y$. Note that the experimental FECs show a force jump $\delta f$ (Fig. 1) when the molecule unfolds or refolds that corresponds to the relaxation of the bead in the trap after the sudden increase or decrease in the RNA extension. To compensate for this effect, we shift the value of the folding forces by an amount equal to $+(-) \delta f / 2$. From (1) and (3) and the unfolding (refolding) force distributions, $\rho_{\mathrm{u}(\mathrm{r})}(f)$, we can extract the effective barrier as

$$
\begin{gathered}
B_{\mathrm{eff}}^{\exp }\left(f-\frac{\delta f}{2}\right)=-\frac{1}{\beta} \ln \left[\frac{\rho_{\mathrm{u}}(f) r}{P_{\mathrm{u}}(f) k_{0}}\right], \\
B_{\mathrm{eff}}^{\exp }\left(f+\frac{\delta f}{2}\right)=G^{0}(N)+g(N, f)-\frac{1}{\beta} \ln \left[\frac{\rho_{\mathrm{r}}(f) r}{P_{\mathrm{r}}(f) k_{0}}\right] .
\end{gathered}
$$

Using polymer theory [12] we can estimate $g(n, f)$ so the expressions (7) and (8) have only two unknown parameters, $G^{0}(N)$ and $k_{0}$. We determine $G^{0}(N)$ by collapsing into a single curve the effective barrier estimates (7) and (8) corresponding to the pulling and relaxing processes at different loading rates. From our data we get $G^{0}(N)=$ $64.5 k_{B} T$ in very good agreement with the Mfold prediction, $G_{\text {Mfold }}^{0}(N)=38 \mathrm{kcal} / \mathrm{mol}=63 k_{B} T$ [15]. In Fig. 2 we show the force-dependent effective barrier obtained using this method. We then determine the value of the attempt frequency $k_{0}$ by fitting (7) and (8) to the prediction by Kramers theory (4). We obtain $k_{0}=10^{5} \mathrm{~s}^{-1}$, which is of 


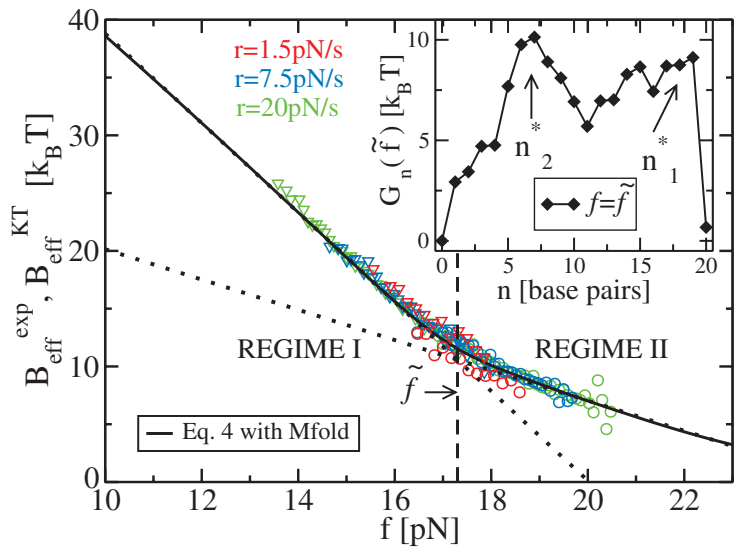

FIG. 2 (color). Effective barrier $B_{\text {eff }}^{\exp }(f)(7)$ and (8) from the pulling (circles) and relaxing (triangles) experimental data compared with the prediction by Kramers theory, $B_{\mathrm{eff}}^{\mathrm{KT}}$ (4) (continuous line). Inset: Free-energy landscape (1) of the RNA hairpin at the crossover force $\tilde{f}=17.3 \mathrm{pN}$.

the order of magnitude of the values reported for other hairpins [19]. The agreement found between the predicted effective barrier (4) and the results from the experiments (7) and (8) validates our description, hence providing a way to estimate the attempt frequency $k_{0}$ [20]. The representation of the effective barrier as a function of the applied force reflects two distinct regimes (Fig. 2) characterized by different slopes of $B_{\text {eff }}(f)$. These correspond to different locations of the effective barrier (5) and different values of the fragility (6). We define a crossover force $\tilde{f}$ as the value at which the extrapolated straight lines corresponding to regimes I and II intersect each other (Fig. 2).

A kinetic barrier is characterized by its location $n^{*}(f)$ and its height $G_{n^{*}}(f)$. As shown in the inset of Fig. 2, the free-energy landscape at force $\tilde{f}$ shows that there are two barriers corresponding to transition states located at $n_{1}^{*} \approx$ $15-19$ and $n_{2}^{*} \approx 6-7$. At low forces, $f<\tilde{f}$ (regime I), the highest barrier is located at $n_{1}^{*}$ and corresponds to the entropy cost associated with the opening of the four bases loop. Whereas for large forces, $f>\tilde{f}$ (regime II), the kinetics is governed by the barrier located at $n_{2}^{*}$ at the interface between the $\mathrm{GC}$ and $\mathrm{AU}$ rich regions of the hairpin. In our experiments we observe the two different regimes, I and II, because the crossover force, $\tilde{f}=$ $17.3 \mathrm{pN}$, is within the experimentally accessible range of rupture forces, $\left[f_{c} \pm 3 \mathrm{pN}\right.$ ] (inset of Fig. 1), where $f_{c}=$ $17.7 \mathrm{pN}$ is the critical force verifying $G_{N}\left(f_{c}\right)=G_{0}\left(f_{c}\right)$ in (1). In order to investigate the unfolding and refolding kinetics over a broader range of forces than those accessible in force-ramp experiments, force-jump experiments [21] could be very helpful. From $B_{\text {eff }}^{\exp }$ and $B_{\text {eff }}^{\mathrm{KT}}$ we obtain the fragility $\mu(f)$ by using (5) and (6). In Fig. 3 we show the agreement between the fragility obtained from the experimentally measured barrier (7) and (8) and Kramers theory (4). Finally, our method can be used to experimentally reconstruct the free-energy landscape of the molecule

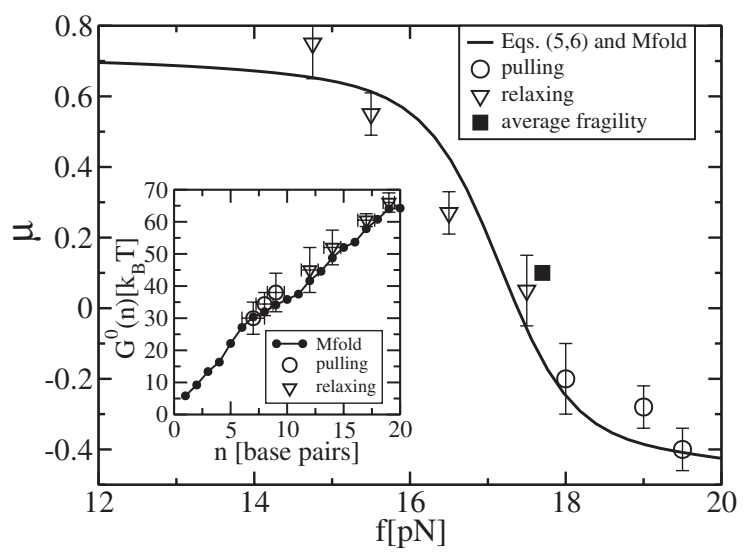

FIG. 3. Fragility of the RNA hairpin as a function of the applied force. The continuous line is the prediction by Kramers theory (5) and (6), whereas symbols correspond to the values obtained from pulling and relaxing data. We also show the average value of the fragility at $f_{c}$ (solid square). Inset: Reconstruction of the zero-force free-energy landscape $G^{0}(n)$ compared to the Mfold prediction [15]. Error bars indicate variability in the estimates obtained for different pulling speeds.

from the sole knowledge of the breakage force distribution at different loading rates. We first determine the location of the force-dependent transition state $n^{*}(f)$ from the measured value of $x_{\text {eff }}^{\mathrm{F}(\mathrm{UF})}(f)(5)$. Using the saddle point approximation we then identify the effective barrier with the largest contribution to the sum appearing in the right-hand side of $(4), B_{\text {eff }}^{\exp }(f) \approx \max _{n} G_{n}(f)=G_{n^{*}}(f)$, where we have taken $h\left(n^{*}\right) \approx 1$ [22]. Finally we apply (1) and extrapolate the free energy $G_{n^{*}}(f)$ to zero force to obtain $G_{n^{*}}(f=0)$. In the inset of Fig. 3 we show the experimentally reconstructed free-energy landscape compared with the results obtained from Mfold [15].

Changes in the position of the transition state (5) along the reaction coordinate axis with the force can correspond to two different situations: (i) The location of the transition state $n^{*}$ does not change with force, but the extension $x_{n}(f)$ does for all configurations as predicted in [23]. (ii) The free-energy landscape of the hairpin shows multiple barriers leading to different transition states depending on the value of the force. This is the case considered in the present study. Interestingly the value of $\mu$ varies with force in the situation (ii) but not in (i). Therefore $\mu$ is a good parameter to identify structural changes in the transition state.

A useful analysis of experimental data for molecular rupture is the two-states model $[24,25]$ where the position of the kinetic barrier along the reaction coordinate is fixed: $x^{\mathrm{F}}, x^{\mathrm{UF}}$, and the fragility, $\tilde{\mu}=\left(x^{\mathrm{F}}-x^{\mathrm{UF}}\right) /\left(x^{\mathrm{F}}+x^{\mathrm{UF}}\right)$, do not depend on the force. In this approximation, $\ln \left\{r\left[\ln \left(1 / P_{\mathrm{u}(\mathrm{r})}\right)\right]\right\}$ is a straight line as a function of the applied force [18]. A plot of the experimental data for $\ln \left\{r\left[\ln \left(1 / P_{\mathrm{u}(\mathrm{r})}\right)\right]\right\}$ versus force displays a nonzero curvature (data not shown) indicating a force-dependent fragility. Moreover, in two-states systems the dependence of the mean value of the unfolding and refolding forces on the 
rate $r$ can be estimated in the experimental regime where $\frac{k_{\mathrm{ur}(\mathrm{r}}\left(f^{\prime}\right) k_{B} T}{r x^{\mathrm{F}(\mathrm{UF})}} \ll 1[26]:$

$$
\langle f\rangle_{\mathrm{u}} \propto \frac{k_{B} T}{x^{\mathrm{F}}}[\ln (r)], \quad\langle f\rangle_{\mathrm{r}} \propto \frac{-k_{B} T}{x^{\mathrm{UF}}}[\ln (r)] .
$$

By fitting the experimental results to (9) we can estimate values for $x^{\mathrm{F}}, x^{\mathrm{UF}}$ which give $\tilde{\mu} \approx 0$. This corresponds to a barrier located in the middle between the F and UF states, in disagreement with the free-energy landscape shown in Fig. 2. Yet, this value coincides with the average fragility measured over the range of forces $f \in[15-19 \mathrm{pN}]$ (Fig. 3), suggesting that fragility estimates obtained by fitting the two-states model to the experimental data correspond to averages of force-dependent fragilities over the range of unfolding and refolding forces explored in the experiments.

We have applied Kramers theory to investigate the kinetics of unfolding and refolding of an RNA hairpin under mechanical force. The analysis of the experimental data for the unfolding and refolding force distributions allows us to determine the location of the force-dependent kinetic barrier, the attempt frequency $k_{0}$ of the hairpin, and the freeenergy landscape of the molecule. The method should be applicable to hairpins with multiple barriers. The theory presented here may fail to describe the unfolding or refolding of the hairpin at low forces and/or high temperatures, where breathing configurations are relevant [23] and the free-energy landscape becomes multidimensional. The presence of force-induced structural changes in molecular transition states is a general feature of biomolecules typically showing a rugged free-energy landscape. Proper consideration of the force dependence of the fragility is crucial to correctly interpret the results from pulling experiments and to relate force unfolding measurements with thermal denaturation experiments.

We are grateful to Bustamante and Tinoco laboratories for kindly providing the facilities where experiments were carried out and to S. B. Smith for technical assistance in the tweezers instrument. M. M. acknowledges a grant from the University of Barcelona. D. C. is supported by NIH Grant No. GM10840 and F. R. by the Ministerio de Educacion y Ciencia in Spain (FIS2004-03545) and Distincio de la Generalitat de Catalunya.

[1] C. Bustamante, Y. R. Chemla, N. R. Forde, and D. Izhaky, Annu. Rev. Biochem. 73, 705 (2004).

[2] M. Carrion-Vazquez et al., Proc. Natl. Acad. Sci. U.S.A. 96, 3694 (1999).

[3] U. Bockelmann, B. Essevaz-Roulet, and F. Heslot, Phys. Rev. Lett. 79, 4489 (1997).

[4] J. Liphardt, B. Onoa, S. B. Smith, I. Tinoco, Jr., and C. Bustamante, Science 292, 733 (2001).

[5] J. Fritz, A. G. Katopodis, F. Kolbinger, and D. Anselmetti, Proc. Natl. Acad. Sci. U.S.A. 95, 12283 (1998).
[6] G. Rubio-Bollinger, S. R. Bahn, N. Agraït, K. W. Jacobsen, and S. Vieira, Phys. Rev. Lett. 87, 026101 (2001).

[7] E. Evans and P. Williams, in Physics of Biomolecules and Cells, Proceedings of the Les Houches Summer School, Session LXXV, edited by H. Flyvberg and F. Julicher (Springer-Verlag, Berlin, 2002).

[8] S. B. Smith, Y. Cui, and C. Bustamante, Methods Enzymol. 361, 134 (2003).

[9] Pulling experiments were performed at $25^{\circ} \mathrm{C}$ in $100 \mathrm{mM}$ Tris $\mathrm{HCl}, 8.1 \mathrm{pH}, 1 \mathrm{~m} M$ ethylenediamine tetraacetic acid with a small interference RNA hairpin that targets the mRNA of the CD4 receptor of the human immunodeficiency virus. For details, see D. Collin et al., Nature (London) 437, 231 (2005).

[10] Experimentally the micropipette is moved at a constant pulling speed. However, above $10 \mathrm{pN}$ (where the molecule typically unfolds and refolds) the loading rate is approximately constant and equal to the stiffness of the trap times the pulling speed. For details, see M. Manosas and F. Ritort, Biophys. J. 88, 3224 (2005).

[11] S. Cocco, R. Monasson, and J. F. Marko, Eur. Phys. J. E 10, 153 (2003).

[12] The mechanical response of the ssRNA, $F_{\text {ssRNA }}(x)$ and $x_{n}(f)$, is described by the worm-like-chain model [13] with a persistence and contour lengths equal to $P_{\text {ssRNA }}=$ $1 \mathrm{~nm}$ and $L_{\mathrm{ssRNA}}=0.59 \mathrm{~nm}$ per base.

[13] C. Bustamante, J. F. Marko, E. G. Siggia, and S. B. Smith, Science 265, 1599 (1994).

[14] D. K. Lubensky and D. R. Nelson, Phys. Rev. E 65, 031917 (2002).

[15] We use the visual oligonucleotide modeling platform from DNA software to predict free-energy values (at $25^{\circ} \mathrm{C}$, in $0.1 \mathrm{M} \mathrm{NaCl})$.

[16] R. Zwanzig, Nonequilibrium Statistical Mechanics (Oxford University Press, New York, 2001), 1st ed., Chap. 4.

[17] The fragility $\mu$ is directly related to the parameter $\alpha$ ( $\mu=$ $2 \alpha-1)$ introduced in J.E. Leffler, Science 117, 340 (1953) to characterize the resemblance of the transition state to the reactant and product of a chemical reaction.

[18] E. Evans and K. Ritchie, Biophys. J. 72, 1541 (1997).

[19] D. Thirumalai and C. Hyeon, Biochemistry 44, 4957 (2005).

[20] $k_{0}$ is not the real attempt frequency of the RNA molecule but has contributions from the setup (handles and bead). Yet these can be shown that do not to change the order of magnitude of its value [M. Manosas et al. (unpublished)].

[21] P. T. X. Li et al., Biophys. J. 90, 250 (2006).

[22] In the experimentally accessible range of rupture forces (around $f_{c}$ ), the condition $G_{n}(f)>0$ for $n=1, \ldots, n^{*}$ is verified. Therefore only the $n^{\prime}=0$ term of the sum appreciably contributes to $h\left(n^{*}\right)$ in (4).

[23] C. Hyeon and D. Thirumalai, Proc. Natl. Acad. Sci. U.S.A. 102, 6789 (2005); Biophys. J. 90, 3410 (2006).

[24] V. Muñoz, P. A. Thompson, J. Hofrichter, and W. A. Eaton, Nature (London) 390, 196 (1997).

[25] F. Ritort, C. Bustamante, and I. N. Tinoco, Jr., Proc. Natl. Acad. Sci. U.S.A. 99, 13544 (2002).

[26] G. Hummer and A. Szabo, Biophys. J. 85, 5 (2003). 